\title{
An unusual case of coarctation of the aorta and a concurrent large-size aortic arch aneurysm in an adult
}

\author{
Wei Huang ${ }^{1}$, Jiayi Song ${ }^{1}$, and Yongjun Qian ${ }^{1}$ \\ ${ }^{1}$ Sichuan University West China Hospital
}

January 20, 2022

\begin{abstract}
We presented a multimodal imaging approach to identifying aortic arch aneurysm with a concurrent coarctation of the aorta in a 52-year-old woman. We successfully adopted a new operative method and described the management of whole process. The detailed imaging and intraoperative data have not been reported in the literature before. This case highlighted the feasibility and successful surgical intervention in a patient with coarctation of the aorta and a concurrent enormous aortic arch aneurysm. An extra-anatomical bypass is an innovative surgical technique that is proved effective and beneficial.
\end{abstract}

Title Page

An unusual case of coarctation of the aorta and a concurrent large-size aortic arch aneurysm in an adult

Wei Huang ${ }^{1}$, MD; Jiayi Song ${ }^{2}$; Yongjun Qian ${ }^{3 \#}, \mathrm{MD}, \mathrm{PhD}$.

${ }^{1}$ Department of Rehabilitation Medicine, West China Hospital, Sichuan University, No. 37 GuoXue Xiang, Chengdu, Sichuan, 610041, People's Republic of China.

${ }^{2}$ West China School of Medicine, West China Hospital, Sichuan University, Chengdu, 610041, People's Republic of China.

${ }^{3}$ Department of Cardiovascular Surgery, West China Hospital, Sichuan University, No. 37 GuoXue Xiang, Chengdu, Sichuan, 610041, People's Republic of China.

\# Address for correspondence: Yongjun Qian

Address: No. 37 GuoXue Xiang, Chengdu, Sichuan, 610041, P.R. China

Phone: +86028 85422897

Fax: +8602885421833

E-mail:qianyongjun@scu.edu.cn

An unusual case of coarctation of the aorta and a concurrent large-size aortic arch aneurysm in an adult

Wei Huang ${ }^{1}$, MD; Jiayi Song ${ }^{2}$; Yongjun Qian ${ }^{3 \#}, \mathrm{MD}, \mathrm{PhD}$.

${ }^{1}$ Department of Rehabilitation Medicine, West China Hospital, Sichuan University, No. 37 GuoXue Xiang, Chengdu, Sichuan, 610041, People's Republic of China. 
${ }^{2}$ West China School of Medicine, West China Hospital, Sichuan University, Chengdu, 610041, People's Republic of China.

${ }^{3}$ Department of Cardiovascular Surgery, West China Hospital, Sichuan University, No. 37 GuoXue Xiang, Chengdu, Sichuan, 610041, People's Republic of China.

\# Address for correspondence: Yongjun Qian

Address: No. 37 GuoXue Xiang, Chengdu, Sichuan, 610041, P.R. China

Phone: +86028 85422897

Fax: +8602885421833

E-mail:qianyongjun@scu.edu.cn

\begin{abstract}
We presented a multimodal imaging approach to identifying aortic arch aneurysm with a concurrent coarctation of the aorta in a 52-year-old woman. We successfully adopted a new operative method and described the management of whole process. The detailed imaging and intraoperative data have not been reported in the literature before. This case highlighted the feasibility and successful surgical intervention in a patient with coarctation of the aorta and a concurrent enormous aortic arch aneurysm. An extra-anatomical bypass is an innovative surgical technique that is proved effective and beneficial.
\end{abstract}

Keywords: Congenital heart disease

A 52-year-old woman was admitted to our department with a high blood pressure of $180 / 70 \mathrm{mmHg}$ measured at the right upper limb, and at both lower limbs, the blood pressure was lower, at 90/50 $\mathrm{mmHg}$. The transthoracic echocardiography revealed a bicuspid aortic valve, moderate aortic valve regurgitation, coarctation of the aorta (COA), and a huge aortic arch aneurysm in close proximity to the left common carotid artery (Figure 1, panels $A$ and B). Computed tomography was performed which confirmed the diagnosis and demonstrated that the left subclavian artery was originated from and deformed by the aneurysm $(5.1 \times 4.8 \mathrm{~cm})$ (Figure 1, panels $C$ and D). Given the findings, surgery was carried out to correct abnormalities. The Bentall procedure was performed to reconstruct the aortic valve and ascending aorta. A 22-mm Dacron graft was used to anastomose to the thoracic descending aorta in an end-to-side fashion while the other end of the dacron graft was anastomosed to the graft in Bentall procedure (Figure 1, panels E and F). Then, part of the aneurysm was excised and the left subclavian artery was ligated.

Diffuse aortic dissection or medial degenerative disease involving the entire aortic arch may result in the development of an exceedingly large aneurysm. ${ }^{[1]}$ On the other hand, COA is a heterogeneous disorder affecting individuals of all age groups. Patients with COA present with varying clinical symptoms, either in isolation or in association with other cardiac defects. ${ }^{[2]}$ Aortic arch aneurysm with a concomitant COA is a rare cardiac malformation and remains a devastating disease associated with underlying serious complications such as sepsis, coagulopathy, and aneurysm rupture. In patients with COA, the outcome is poor without surgical correction. The mean age of death was 34 years, with the mortality rate reaching $75 \%$ at 43 years of age. ${ }^{[3]}$ Since the first surgical intervention for correcting the COA described by Crafoord ${ }^{[4]}$ in 1944 , advancements have been made in surgical techniques to improve the quality of treatment and the outcome of patients with this disorder. Despite improvements in the mortality and morbidity rates, operations for the COA continue to carry significant risks of adverse events or patient morbidity. Concomitant aortic arch aneurysm combined with COA is extremely rare. Therefore, individualized patient assessment and careful plans for the most suitable treatment approach are required before on embarking a high-risk surgery. Our case highlighted the feasibility and successful surgical intervention in a patient with COA and a concurrent enormous aortic arch aneurysm. An extra-anatomical bypass was performed which proved beneficial in this case.

\title{
CONFLICT OF INTERESTS
}


The authors declare that there are no conflict of interests.

\section{ETHICS STATEMENT}

This manuscript and all of its content meet the ethical guidelines, including adherence to the legal requirements of the study country. The need for patient consent was waived.

\section{FUNDING}

This study was funded by 1.3.5 project for disciplines of excellence-Clinical Research Incubation Project, West China Hospital, Sichuan University (ZYJC21064).

\section{References}

1. Patel, H.J., Deeb, G.M. Ascending and arch aorta pathology, natural history, and treatment. Circulation. 2008; 118 (2):188-195.

2. Rachel D Torok, Michael J Campbell, Gregory A Fleming, Kevin D Hill. Coarctation of the aorta: Management from infancy to adulthood [J]. World Journal of Cardiology, 2015; 7(11):765-775.

3. Campbell M. Natural history of coarctation of the aorta. Br Heart J 1970; 32: 633-640 [PMID: 5470045 DOI: $10.1136 /$ hrt.32.5.633]

4. Kvitting JP, Olin CL. Clarence Crafoord: a giant in cardiothoracic surgery, the first to repair aortic coarctation. Ann Thorac Surg 2009;87:342-346 [PMID:19101336 DOI: 10.1016/j.athoracsur.2008.08. 072]

\section{Figures}

Figure 1. Panels A\&B, the transthoracic echocardiography revealed a bicuspid aortic valve, moderate aortic valve regurgitation (arrow), coarctation of the aorta, and a huge aortic arch aneurysm in close proximity to the left common carotid artery. Panels C\&D, computed tomography and three-dimensional reconstruction demonstrated that the left subclavian artery was originated from and deformed by the aneurysm. Panels $\mathrm{E} \& \mathrm{~F}$, Dacron graft (asterisk) was used to anastomose to the thoracic descending aorta in an end-to-side fashion, the other end of the Dacron graft was anastomosed to the graft in Bentall procedure. (LV, left ventricle; LA, left atrium; CoA, coarctation of aorta; Aneu, aneurysm; AA, ascending aorta; DA, descending aorta; BPG, bypass graft.) 

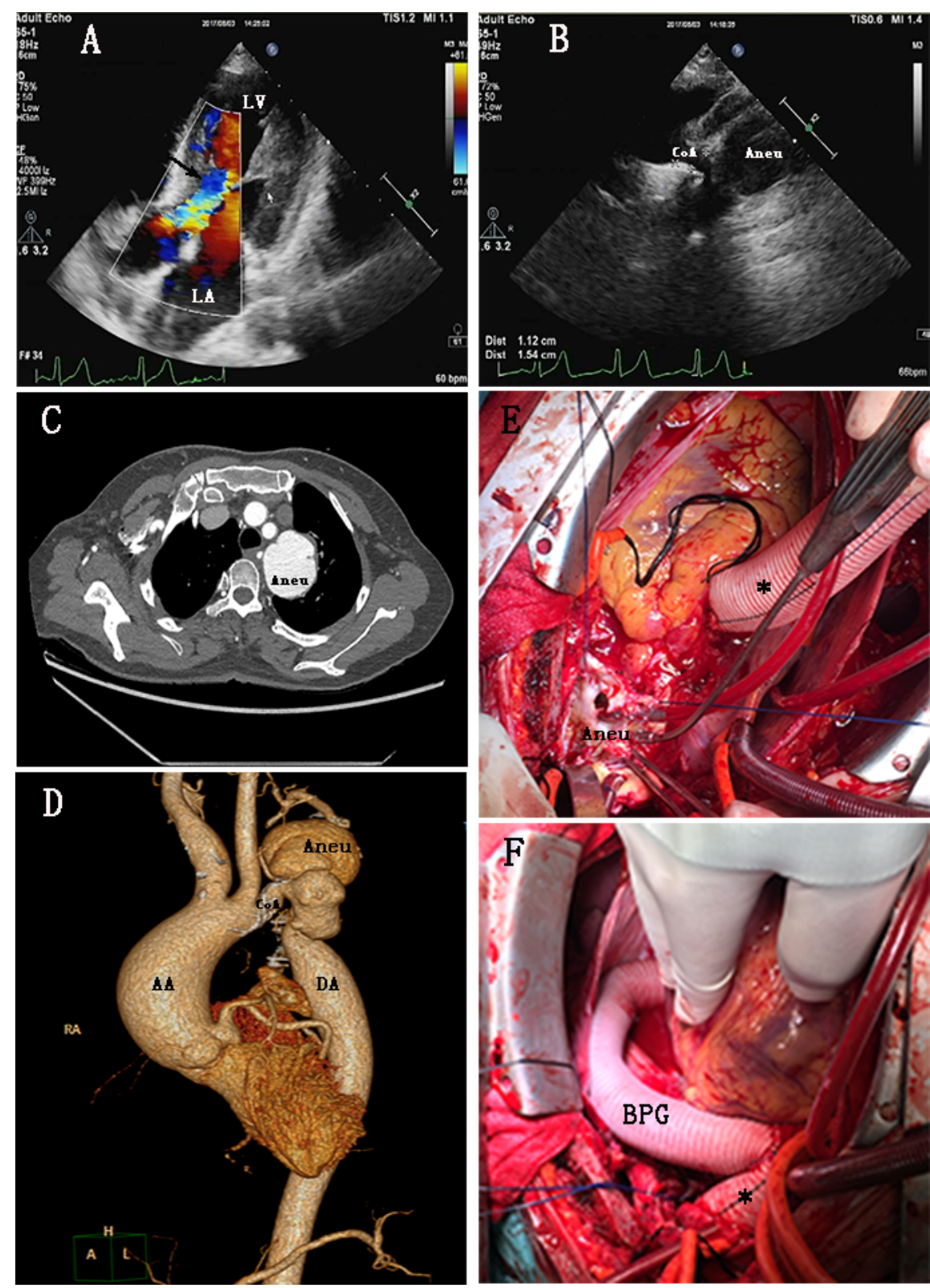